\title{
L'infirmier(ère) seul(e) devant une situation de détresse médicale : des recommandations professionnelles pour préciser son rôle hors présence médicale
}

\author{
The nurse alone in front of a medical distress situation: professional recommendations to specify \\ its role in the absence of professional medical support
}

\author{
J. Schmidt \\ (C) SFMU et Springer-Verlag France 2012
}

Les situations de détresse médicale inopinée, engageant le pronostic vital ou fonctionnel, imposent des soins d'urgences immédiats par des gestes spécifiques afin de préserver la vie et/ou la fonction des victimes. L'aide médicale urgente en France apporte une réponse adaptée grâce aux modalités organisationnelles de notre système de santé permettant une médicalisation préhospitalière de qualité précédent la réponse médicale hospitalière. Cette organisation ne peut toutefois ignorer l'obligation d'apporter une réponse immédiate et adaptée aux situations de détresse médicale auxquelles sont confrontées les infirmiers diplômés d'état (IDE) au quotidien dans leur exercice professionnel. On pense alors en premier lieu aux IDE assurant un transport infirmier interhospitalier ou exerçant leur métier comme infirmier sapeur pompier volontaire. Ces deux situations ne doivent pas faire ignorer les situations de détresse médicale confrontant les IDE des établissements d'hébergements des personnes âgées dépendantes, les IDE en milieu scolaire ou en médecine du travail, les IDE militaires, etc.

La rédaction de recommandations par la Société française de médecine d'urgence (SFMU) sous la direction d'Yvon Croguennec, président de la Commission soins et urgences de notre société savante et sous la responsabilité de Dominique Pateron, ancien président de la SFMU, en étroite collaboration avec nos partenaires de la Société française d'anesthésie et de réanimation (SFAR), du Collège français de réanimation cardiopulmonaire (CFRC) et du Club des anesthésistes-réanimateurs et urgentistes militaires

\footnotetext{
J. Schmidt $(\bowtie)$

Pôle Samu-Smur-urgences, CHU de Clermont-Ferrand, 58, rue Montalembert, F-63003 Clermont-Ferrand cedex 01, France

e-mail : e-mail : jschmidt@chu-clermontferrand.fr

Faculté de Médecine, Université d'Auvergne Clermont 1, 28, place Henri-Dunant, F-6300 BP 38 Clermont-Ferrand cedex 1, France
}

(CARUM), apporte des réponses à 18 situations fréquentes et identifiées d'urgences [1]. Ces situations ont été retenues sur le principe qu'une prise en charge immédiate permettait d'envisager un bénéfice clinique pour le patient dans l'attente de l'intervention d'une équipe SMUR. Les réponses apportées s'inscrivent dans le cadre règlementaire de l'exercice de la fonction d'IDE [2] et sont fondées sur les publications scientifiques existantes, encore trop rares et de niveaux de preuve peu élevées.

Toutefois, ces recommandations ne représentent que la première pierre à l'édifice « Médecine d'urgence » et précisent, à la lecture attentive des situations cliniques retenues, les conditions de réalisation et de contrôle des gestes et soins spécifiques que l'IDE peut effectuer. Ainsi, définir les modalités de gestion des situations de détresse médicale prises en charge par l'infirmier sans médecin, représente un complément des dispositifs existants et devrait éviter une hétérogénéité de pratique et certains excès constatés liés à l'absence de référentiel. Le texte rappelle que l'application de ces recommandations ne sera qu'une première étape du soin attendu et ne se substitue pas mais s'intègre dans l'organisation des secours à la personne et de l'aide médicale urgente telle qu'elle existe en France. Celle-ci est fondée sur le recours permanent possible de l'avis du médecin régulateur du SAMU et sur l'intervention d'une équipe médicalisée en cas de besoin. La mise en place des recommandations imposera toutefois dans le futur de gros efforts d'investissement en termes de formation initiale des IDE au sein des instituts de formations en soins infirmiers (IFSI), et dans l'immédiat, la mise sur pied de sessions de formations qui pourraient s'appuyer sur les centres d'enseignement des soins d'urgence (CESU). La SFMU veillera aux côtés de ses partenaires à l'application de ces nouveaux outils. Elle encourage dès à présent leur évaluation par des protocoles de recherche infirmiers menés dans le respect de leur périmètre. 
Je souhaite, au nom de tous les acteurs de la médecine d'urgence, remercier le comité d'organisation, les experts et les nombreux relecteurs critiques de ces recommandations. Un grand merci plus particulièrement à Yvon Croguennec et à Dominique Pateron.

Une nouvelle pierre est posée, la construction du mur se poursuit.

\section{Références}

1. Croguennec Y, Braun F, Ricard-Hibon A, et al (2012) Recommandations professionnelles: l'infirmier(ère) diplômé(e) d'état seul(e) devant une situation de détresse médicale. Ann Fr Med Urg 2:5769

2. Décret $n^{\circ} 2004-802$ du 29 juillet 2004 relatif aux parties IV et V (dispositions réglementaires) du code de la santé publique et modifiant certaines dispositions de ce code. (http://www.legifrance. gouv.fr/affichTexte.do?

cidTexte $=$ JORFTEXT000000787339\&dateTexte $=$ ) 